\title{
Stability analysis of second-order differential systems with Erlang distribution random impulses
}

\author{
Shuorui Zhang and Jitao Sun ${ }^{*}$
}

"Correspondence: sunjt@sh163.net Department of Mathematics, Tongji University, Shanghai, 200092, China

\begin{abstract}
Differential systems with random impulses are a new kind of mathematical models. In this paper, we put forward a model of second-order impulsive differential systems with Erlang distribution random impulses. Sufficient conditions are obtained for oscillation in mean and $p$-moment stability of this model respectively. An example is presented to illustrate the efficiency of the results obtained.
\end{abstract}

MSC: 34A37; 34A12; 34A34

Keywords: linear differential system; random impulses; stability; oscillation; Erlang distribution

\section{Introduction}

It is recognized that the impulsive differential system is an effective model for many real world phenomena, thus it has been widely used in the study of physics, engineering, information and communications technology, etc. in the past years and a lot of valuable results have been obtained (see [1-15] and references therein).

For impulsive differential systems, most researchers concern about two kinds of impulse times: fixed impulse times and varying impulse times, which mean that the impulse time is some functions of the 'state $x$ ' [7-9]. However, the impulse phenomena sometimes happen at random times, and any solution of systems driven by this kind of impulses is a stochastic process, which is very different from those of differential systems with impulses at fixed moments and varying impulse times [11]. Thus, the randomness introduced in impulsive differential systems by this way has brought us new difficulties and problems in the study of impulsive differential systems. Some other kinds of randomness brought to a system can be seen in [16-18].

In fact, only few researchers have studied this kind of impulse (see [11-15] and references therein). Wu and Meng first introduced random impulsive ordinary differential equations and investigated the boundedness of solutions to these models by Lyapunov's direct method in [12]. In [13], Wu et al. discussed the existence and uniqueness in mean square of solutions to certain impulsive differential systems by employing the Cauchy-Schwarz inequality, Lipschitz condition and techniques in stochastic analysis. In [14], Anguraj et al. presented the existence and exponential stability of mild solutions of semilinear differential equations with random impulses. In [15], the existence and uniqueness of stochastic differential equations with random impulses were studied by Wu and Zhou via employing 
the Bihari inequality under non-Lipschitz conditions. In [11], Wu and Duan studied the oscillation, stability and boundedness of second-order differential systems with random impulses. But in their paper, the random impulses must be independent and follow the same exponential distribution which is a very strong condition. Inspired by their work, we generalized their results to a much more general distribution, called the Erlang distribution, which has been widely used to describe the waiting times. We will call this kind of impulse the 'random impulses' throughout this paper. And in this paper, we will discuss properties such as oscillation, stability of second-order differential systems with Erlang distribution random impulses.

The rest of this paper is organized as follows. In Section 2, we recall some preliminary definitions. In Section 3, we first give some useful and important lemmas, then establish our main oscillation and stability results. In Section 4, we give an example to illustrate the effectiveness of our results. Finally, the conclusion and the acknowledgements are mentioned in Section 5.

\section{Preliminaries}

Let $(\Omega, \mathcal{F}, \mathbb{P})$ be a given complete probability space and $\tau_{k}$ 's be non-negative random variables defined in $(\Omega, \mathcal{F}, \mathbb{P}), k=1,2, \ldots$ Furthermore, assume that $\tau_{i}$ and $\tau_{j}$ are independent with each other when $i \neq j$ for $i, j=1,2, \ldots \mathbb{E}$ denotes the mathematical expectation. For the sake of simplicity, we denote $\mathbb{N}=\{1,2, \ldots\}, \mathbb{R}=(-\infty,+\infty), \mathbb{R}_{+}=[0,+\infty), \mathbb{R}_{\tau}=[\tau,+\infty)$, where $\tau \in \mathbb{R}$ is a constant. Consider the second-order linear differential systems with random impulses,

$$
y^{\prime \prime}(t)+a(t) y^{\prime}(t)+p(t) y(t)=0, \quad t \in \mathbb{R}_{\tau}, t \neq \xi_{k}, \forall k \in \mathbb{N},
$$

and

$$
\triangle y\left(\xi_{k}\right)=b_{k} y\left(\xi_{k}^{-}\right), \quad \Delta y^{\prime}\left(\xi_{k}\right)=b_{k} y^{\prime}\left(\xi_{k}^{-}\right), \quad \forall k \in \mathbb{N},
$$

where $a, p \in\left(\mathbb{R}_{\tau}, \mathbb{R}\right)$ are Lebesgue measurable and locally essentially bounded functions, $\xi_{0}=t_{0} \in \mathbb{R}_{\tau}$ and $\xi_{k}=\xi_{k-1}+\tau_{k}$ for all $k \in \mathbb{N}, \Delta y\left(\xi_{k}\right) \equiv y\left(\xi_{k}\right)-y\left(\xi_{k}^{-}\right), \Delta y^{\prime}\left(\xi_{k}\right) \equiv y^{\prime}\left(\xi_{k}\right)-y^{\prime}\left(\xi_{k}^{-}\right)$, and $y\left(\xi_{k}^{-}\right)=\lim _{t \rightarrow \xi_{k}^{-}} y(t)$.

Before giving the main results, we first introduce some definitions.

Definition 2.1 A stochastic process $y(t)$ is said to be a sample path solution to the system (1) with (2) satisfying the initial value condition,

$$
y_{t_{0}}=y_{0},
$$

if for any sample value $t_{1}<t_{2}<\cdots<t_{k}<\cdots$, of $\left\{\xi_{k}\right\}_{k \geq 1}$, then $y(t)$ satisfies the following equations:

$$
\left\{\begin{array}{l}
y^{\prime \prime}(t)+a(t) y^{\prime}(t)+p(t) y(t)=0, \quad t \in \mathbb{R}_{\tau}, \xi_{k} \neq t, \forall k \in \mathbb{N}, \\
\triangle y\left(t_{k}\right)=b_{k} y\left(t_{k}^{-}\right), \quad \forall k \in \mathbb{N}, \\
\triangle y^{\prime}\left(t_{k}\right)=b_{k} y^{\prime}\left(t_{k}^{-}\right), \quad \forall k \in \mathbb{N} .
\end{array}\right.
$$

Definition 2.2 Let $p>0$, then the system (1) with (2) is said to be 
(i) $p$-moment stable if for any $\epsilon>0$ and $t_{0} \in \mathbb{R}_{\tau}$, there exists a $\delta=\delta\left(\epsilon, t_{0}\right)>0$ such that

$$
\left|y_{0}\right|^{p}<\delta \quad \text { implies } \quad \mathbb{E}|y(t)|^{p}<\epsilon \quad \text { for all } t \geq t_{0}
$$

(ii) uniformly $p$-moment stable if the $\delta$ in (i) is independent of $t_{0}$;

(iii) asymptotically $p$-moment stable if it is $p$-moment stable, and for any $\epsilon_{1}>0, \delta_{1}$ and $t_{0} \in \mathbb{R}_{\tau}$, there exists a $T=T\left(\epsilon_{1}, \delta_{1}, t_{0}\right)$ such that

$$
\left|y_{0}\right|^{p}<\delta_{1} \quad \text { implies } \quad \mathbb{E}|y(t)|^{p}<\epsilon_{1} \quad \text { for all } t \geq t_{0}+T
$$

(iv) uniformly asymptotically $p$-moment stable if it is uniformly $p$-moment stable, and the $T$ in (iii) is independent of $t_{0}$.

Generally, two-moment stable is called stable in mean square.

Definition 2.3 A solution $y(t)$ to the system (1) with (2) is said to be non-oscillatory in mean if $\mathbb{E} y(t)$ is either eventually positive or eventually negative. Otherwise, it is called oscillatory.

Definition 2.4 The Erlang distribution is a continuous probability distribution with probability density function as follows:

$$
f(x ; k, \lambda)= \begin{cases}\frac{\lambda^{k} x^{k-1} e^{-\lambda x}}{(k-1) !}, & x>0, \\ 0, & \text { otherwise }\end{cases}
$$

where $k \in \mathbb{N}$ is the shape parameter and $\lambda>0$ is the rate parameter. In the following, we will denote the probability density function of the Erlang distribution by $\operatorname{Erlang}(k, \lambda)$.

Remark 2.1 Some properties of the Erlang distribution.

(i) The mean value of $\operatorname{Erlang}(k, \lambda)$ is $\frac{k}{\lambda}$, the variance of $\operatorname{Erlang}(k, \lambda)$ is $\frac{k}{\lambda^{2}}$;

(ii) $\operatorname{Erlang}(1, \lambda)=\operatorname{exponential}(\lambda)$;

(iii) If $X, Y$ are independent, and $X \sim \operatorname{Erlang}\left(k_{1}, \lambda\right), Y \sim \operatorname{Erlang}\left(k_{2}, \lambda\right)$, then $X+Y \sim \operatorname{Erlang}\left(k_{1}+k_{2}, \lambda\right)$

(iv) The cumulative distribution function (CDF) of the Erlang distribution is

$$
F(x ; k, \lambda)=1-\sum_{n=0}^{k-1} e^{-\lambda x} \frac{(\lambda x)^{n}}{n !} .
$$

Consider the following auxiliary differential system:

$$
x^{\prime \prime}(t)+a(t) x^{\prime}(t)+p(t) x(t)=0, \quad t \in \mathbb{R}_{\tau} .
$$

A solution $x(t)$ to the system (3) means that $x(t)$ has the second-order derivative $x^{\prime \prime}(t)$ on $\mathbb{R}_{\tau}$ and satisfies the system (3) for all $t \in \mathbb{R}_{\tau}$.

The following condition will be needed by all main results in Section 3.

(H) Let $\tau_{k}$ follow Erlang $\left(m_{k}, \lambda\right)$, where $m_{k} \in \mathbb{N}, k=1,2, \ldots$, and $m_{i} \leq m_{j}$, for any $i \leq j$ and let $\tau_{i}$ be independent with $\tau_{j}$ when $i \neq j$. 
Remark 2.2 From Remark 2.1(i), we know that the assumption $m_{i} \leq m_{j}(i \leq j)$ means that the impulses interval $\tau_{k}$ is non-decreasing in $k$ in the sense of expectation.

\section{Main results}

In this section, some results on $p$-moment stability and oscillation in mean of the secondorder linear differential systems (1) with random impulses (2) are presented. Inspired by [19], we obtain the following lemma, which guarantees $\lim _{k \rightarrow+\infty} \xi_{k}=+\infty$ with probability 1.

Lemma 3.1 Assume that the condition $(\mathrm{H})$ holds, then $\lim _{k \rightarrow+\infty} \xi_{k}=+\infty$ with probability 1 .

Proof For a given non-negative random variable $\tau$ and a constant $c>0$, we define

$$
\tau^{(c)}:= \begin{cases}\tau, & \text { if } \tau \leq c \\ 0, & \text { otherwise. }\end{cases}
$$

By Kolmogorov's three series theorem and Kolmogorov's zero-or-one laws [20], $\sum_{k=1}^{+\infty} \tau_{k}=$ $+\infty$ almost surely if and only if at least one of the series $\sum_{k=1}^{+\infty} \mathbb{P}\left(\tau_{k}>c\right)$ and $\sum_{k=1}^{+\infty} \mathbb{E}\left(\tau_{k}^{(c)}\right)$ diverges for some $c>0$ or any $c>0$.

From the CDF of the Erlang distribution, we obtain that $\mathbb{P}\left(\tau_{k}>c\right)=1-\mathbb{P}\left(\tau_{k} \leq c\right)=1-$ $F\left(c ; m_{k}, \lambda\right)$ is increasing in $k$, together with $\mathbb{P}\left(\tau_{1}>c\right)>0$, we conclude that $\sum_{k=1}^{+\infty} \mathbb{P}\left(\tau_{k}>c\right)$ diverges. Thus, we know that $\sum_{k=1}^{+\infty} \tau_{k}=+\infty$ almost surely. The proof is complete.

Lemma 3.2 (see [11]) $y(t)$ is a solution of the system (1) with (2) if and only if

$$
y(t)=\sum_{k=0}^{+\infty}\left(\prod_{i=1}^{k}\left[1+b_{i}\right] \cdot \mathcal{X}_{\left[\xi_{k}, \xi_{k+1}\right)}(t)\right) x(t)
$$

where $x(t)$ is a solution of the system (3) with the same initial conditions of the system (1) with (2), and $\mathcal{X}$ is the index function, i.e.,

$$
\mathcal{X}_{\left[\xi_{k}, \xi_{k+1}\right)}(t)= \begin{cases}1, & \text { if } \xi_{k} \leq t<\xi_{k+1} \\ 0, & \text { otherwise. }\end{cases}
$$

Here and in the sequel, we assume that a product equals unity if the number of factors is equal to zero.

Theorem 3.1 Let the condition $(\mathrm{H})$ hold. Further assume that there are a finite number of $b_{k}$ such that $b_{k}<-1$. If there exists a $T \in \mathbb{R}_{\tau}$ such that

$$
\sum_{k=0}^{+\infty}\left(\prod_{i=1}^{k}\left(1+b_{i}\right) \cdot \sum_{n=m_{1}+m_{2}+\cdots+m_{k}}^{m_{1}+m_{2}+\cdots+m_{k+1}-1} \frac{\left(\lambda z_{0}\right)^{n}}{n !}\right)
$$

does not change its sign for all $t \geq T$, then all solutions of the system (1) with (2) are oscillatory in mean if and only if all solutions of the system (3) are oscillatory. Here and in the following, $z_{0} \equiv t-t_{0}$. 
Proof Let $y(t)$ be any sample path solution of the system (1) with (2), then it follows from Lemma 3.2 that

$$
y(t)=\sum_{k=0}^{+\infty}\left(\prod_{i=1}^{k}\left(1+b_{i}\right) \cdot \mathcal{X}_{\left[\xi_{k}, \xi_{k+1}\right)}(t)\right) x(t) .
$$

Since there are a finite number of $b_{k}$ such that $b_{k}<-1$, there exists a $K$ such that $\prod_{i=1}^{k}\left(1+b_{i}\right)$ are either non-negative or non-positive for all $k \geq K$. Hence, by either monotone convergence or Tonelli's theorem [20],

$$
\begin{aligned}
\mathbb{E} y(t) & =\mathbb{E} \sum_{k=0}^{+\infty}\left(\prod_{i=1}^{k}\left(1+b_{i}\right) \cdot \mathcal{X}_{\left[\xi_{k}, \xi_{k+1}\right)}(t)\right) x(t) \\
& =\sum_{k=0}^{+\infty} \mathbb{E}\left(\prod_{i=1}^{k}\left(1+b_{i}\right) \cdot \mathcal{X}_{\left[\xi_{k}, \xi_{k+1}\right)}(t)\right) x(t) \\
& =\sum_{k=0}^{+\infty}\left(\prod_{i=1}^{k}\left(1+b_{i}\right) \cdot \mathbb{E} \mathcal{X}_{\left[\xi_{k}, \xi_{k+1}\right)}(t)\right) x(t) .
\end{aligned}
$$

Further,

$$
\begin{aligned}
\mathbb{E} \mathcal{X}_{\left[\xi_{k}, \xi_{k+1}\right)}(t)= & \mathbb{P}\left(\tau_{1}+\tau_{2}+\cdots+\tau_{k} \leq z_{0}<\tau_{1}+\tau_{2}+\cdots+\tau_{k+1}\right) \\
& =\mathbb{P}\left(\tau_{1}+\tau_{2}+\cdots+\tau_{k} \leq z_{0}\right)-\mathbb{P}\left(\tau_{1}+\tau_{2}+\cdots+\tau_{k+1} \leq z_{0}\right) \\
& =\sum_{n=m_{1}+m_{2}+\cdots+m_{k}}^{m_{1}+m_{2}+\cdots+m_{k+1}-1} \frac{e^{-\lambda z_{0}}\left(\lambda z_{0}\right)^{n}}{n !} .
\end{aligned}
$$

So,

$$
\begin{aligned}
\mathbb{E} y(t) & =\sum_{k=0}^{+\infty}\left(\prod_{i=1}^{k}\left(1+b_{i}\right) \cdot \sum_{n=m_{1}+m_{2}+\cdots+m_{k}}^{m_{1}+m_{2}+\cdots+m_{k+1}-1} \frac{e^{-\lambda z_{0}}\left(\lambda z_{0}\right)^{n}}{n !}\right) x(t) \\
& =e^{-\lambda z_{0}} x(t) \sum_{k=0}^{+\infty}\left(\prod_{i=1}^{k}\left(1+b_{i}\right) \cdot \sum_{n=m_{1}+m_{2}+\cdots+m_{k}}^{m_{1}+m_{2}+\cdots+m_{k+1}-1} \frac{\left(\lambda z_{0}\right)^{n}}{n !}\right) .
\end{aligned}
$$

Since

$$
\sum_{k=0}^{+\infty}\left(\prod_{i=1}^{k}\left(1+b_{i}\right) \cdot \sum_{n=m_{1}+m_{2}+\cdots+m_{k}}^{m_{1}+m_{2}+\cdots+m_{k+1}-1} \frac{\left(\lambda z_{0}\right)^{n}}{n !}\right)
$$

does not change its sign for all $t \geq T$,

$$
e^{-\lambda z_{0}} x(t) \sum_{k=0}^{+\infty}\left(\prod_{i=1}^{k}\left(1+b_{i}\right) \cdot \sum_{n=m_{1}+m_{2}+\cdots+m_{k}}^{m_{1}+m_{2}+\cdots+m_{k+1}-1} \frac{\left(\lambda z_{0}\right)^{n}}{n !}\right)
$$

does not change its sign for all $t \geq T$, too. Hence, $\mathbb{E} y(t)$ has the same sign as $x(t)$ for all $t \geq T$. That is, all solutions of the system (1) with (2) are oscillatory in mean if and only if all solutions of the system (3) are oscillatory. The proof is complete. 
Theorem 3.2 Let the condition $(\mathrm{H})$ hold and further assume that there are a finite number of $b_{k}$ such that $b_{k}<-1$. Then all solutions of the system (1) with (2) are oscillatory in mean if and only if all solutions of the system (3) are oscillatory.

Proof According to Theorem 3.1, we only need to prove that there exists a $T \in \mathbb{R}_{\tau}$ such that

$$
\sum_{k=0}^{+\infty}\left(\prod_{i=1}^{k}\left(1+b_{i}\right) \cdot \sum_{n=m_{1}+m_{2}+\cdots+m_{k}}^{m_{1}+m_{2}+\cdots+m_{k+1}-1} \frac{\left(\lambda z_{0}\right)^{n}}{n !}\right)
$$

does not change its sign for all $t \geq T$.

In the following, we will discuss the sign of (4) in two cases respectively.

Case I. Assume that there are a finite number of $b_{k}$ such that $b_{k}<-1$ and no $b_{i}=-1$. Then there exists a finite set $\hat{N}=\left\{k_{i}: k_{i} \in \mathbb{N}\right.$ satisfying $k_{1}<k_{2}<\cdots<k_{n}$, where $n$ is finite $\}$ such that $b_{k}>-1$ for all $k \in \mathbb{N} \backslash \hat{N}$ and $b_{k}<-1$ for all $k \in \hat{N}$.

(a) If $n$ is odd,

$$
\prod_{i=1}^{k}\left(1+b_{i}\right)<0 \quad \text { for all } k \geq k_{n}
$$

So,

$$
\prod_{i=1}^{k}\left(1+b_{i}\right) \cdot \sum_{n=m_{1}+\cdots+m_{k}}^{m_{1}+\cdots+m_{k+1}-1} \frac{\left(\lambda z_{0}\right)^{n}}{n !}<0 \quad \text { for all } k \geq k_{n} \text { and } t>t_{0} .
$$

For any fixed $k, 0 \leq k<k_{n}$,

$$
\begin{aligned}
& \prod_{i=1}^{k_{n}}\left|1+b_{i}\right| \frac{\left(\lambda z_{0}\right)^{m_{1}+\cdots+m_{k_{n}}+j-1}}{\left(m_{1}+\cdots+m_{k_{n}}+j-1\right) !} \\
& \quad \geq \prod_{i=k+1}^{k_{n}}\left|1+b_{i}\right| \frac{\left(\lambda z_{0}\right)^{m_{k+1}+\cdots+m_{k_{n}}}}{\left(m_{1}+\cdots+m_{k_{n}}+j-1\right) !} \cdot \prod_{i=1}^{k}\left|1+b_{i}\right| \frac{\left(\lambda z_{0}\right)^{m_{1}+\cdots+m_{k}+j-1}}{\left(m_{1}+\cdots+m_{k}+j-1\right) !} \\
& \quad \geq \prod_{i=k+1}^{k_{n}}\left|1+b_{i}\right| \frac{\left(\lambda z_{0}\right)^{m_{k+1}+\cdots+m_{k_{n}}}}{\left(m_{1}+\cdots+m_{k_{n}}+m_{k+1}-1\right) !} \cdot \prod_{i=1}^{k}\left|1+b_{i}\right| \frac{\left(\lambda z_{0}\right)^{m_{1}+\cdots+m_{k}+j-1}}{\left(m_{1}+\cdots+m_{k}+j-1\right) !}
\end{aligned}
$$

holds for $j=1,2, \ldots, m_{k+1}$.

In fact,

$$
\prod_{i=k+1}^{k_{n}}\left|1+b_{i}\right| \frac{\left(\lambda z_{0}\right)^{m_{k+1}+\cdots+m_{k_{n}}}}{\left(m_{1}+\cdots+m_{k_{n}}+m_{k+1}-1\right) !}=\beta_{k} \cdot z_{0}^{m_{k+1}+\cdots+m_{k_{n}}} \geq k_{n}-1
$$

holds for all $t \geq t_{0}+T_{k}$, where $T_{k}=\max \left\{1, \frac{k_{n}-1}{\beta_{k}}\right\}$, and

$$
\beta_{k}=\prod_{i=k+1}^{k_{n}}\left|1+b_{i}\right| \frac{\lambda^{m_{k+1}+\cdots+m_{k_{n}}}}{\left(m_{1}+\cdots+m_{k_{n}}+m_{k+1}-1\right) !}
$$

is a positive constant. 
Thus,

$$
\begin{aligned}
& \prod_{i=1}^{k_{n}}\left|1+b_{i}\right| \frac{\left(\lambda z_{0}\right)^{m_{1}+\cdots+m_{k_{n}}+j-1}}{\left(m_{1}+\cdots+m_{k_{n}}+j-1\right) !} \\
& \quad \geq\left(k_{n}-1\right) \prod_{i=1}^{k}\left|1+b_{i}\right| \frac{\left(\lambda z_{0}\right)^{m_{1}+\cdots+m_{k}+j-1}}{\left(m_{1}+\cdots+m_{k}+j-1\right) !}
\end{aligned}
$$

holds for $j=1,2, \ldots, m_{k+1}$. From (6), we obtain that

$$
\left|\prod_{i=1}^{k_{n}}\left(1+b_{i}\right) \cdot \sum_{n=m_{1}+\cdots+m_{k_{n}}}^{m_{1}+\cdots+m_{k_{n}+1^{-1}}} \frac{\left(\lambda z_{0}\right)^{n}}{n !}\right| \geq\left(k_{n}-1\right)\left|\prod_{i=1}^{k}\left(1+b_{i}\right) \cdot \sum_{n=m_{1}+\cdots+m_{k}}^{m_{1}+\cdots+m_{k+1}-1} \frac{\left(\lambda z_{0}\right)^{n}}{n !}\right| .
$$

From (5) and (7), it follows that

$$
\sum_{k=0}^{+\infty}\left(\prod_{i=1}^{k}\left(1+b_{i}\right) \cdot \sum_{n=m_{1}+m_{2}+\cdots+m_{k}}^{m_{1}+m_{2}+\cdots+m_{k+1}-1} \frac{\left(\lambda z_{0}\right)^{n}}{n !}\right)<0
$$

for $t \geq t_{0}+T$, where $T=\max _{0 \leq k<k_{n}} T_{k}$.

(b) If $n$ is even, similar to the procedure of (a) in Case I, we can prove that

$$
\sum_{k=0}^{+\infty}\left(\prod_{i=1}^{k}\left(1+b_{i}\right) \cdot \sum_{n=m_{1}+m_{2}+\cdots+m_{k}}^{m_{1}+m_{2}+\cdots+m_{k+1}-1} \frac{\left(\lambda z_{0}\right)^{n}}{n !}\right)>0
$$

holds for all $t \geq t_{0}+T$.

From (a), (b), we know that

$$
\sum_{k=0}^{+\infty}\left(\prod_{i=1}^{k}\left(1+b_{i}\right) \cdot \sum_{n=m_{1}+m_{2}+\cdots+m_{k}}^{m_{1}+m_{2}+\cdots+m_{k+1}-1} \frac{\left(\lambda z_{0}\right)^{n}}{n !}\right)
$$

does not change its sign for all $t \geq t_{0}+T$.

Case II. Assume there are a finite number of $b_{k}$ such that $b_{k}<-1$ and at least a $b_{i}=$ -1 . Then let $m=\min \left\{i \in \mathbb{N}: b_{i}=-1\right\}$, and let $b_{k}>-1$ for all $k \in\{1,2, \ldots, m-1\} \backslash \hat{N}$ and $b_{i}<-1$ for all $i \in \hat{N}$, where $\hat{N}=\left\{k_{1}, k_{2}, \ldots, k_{n}\right\}$ satisfying $k_{1}<k_{2}<\cdots<k_{n}$. Without loss of generality, we assume $k_{n}<m$. Then

$$
\prod_{i=1}^{k}\left(1+b_{i}\right)=0 \quad \text { for all } k \geq m
$$

Thus,

$$
\begin{aligned}
\sum_{k=0}^{+\infty} & \left(\prod_{i=1}^{k}\left(1+b_{i}\right) \cdot \sum_{n=m_{1}+m_{2}+\cdots+m_{k}}^{m_{1}+m_{2}+\cdots+m_{k+1}-1} \frac{\left(\lambda z_{0}\right)^{n}}{n !}\right) \\
= & \sum_{k=0}^{m-1}\left(\prod_{i=1}^{k}\left(1+b_{i}\right) \cdot \sum_{n=m_{1}+m_{2}+\cdots+m_{k}}^{m_{1}+m_{2}+\cdots+m_{k+1}-1} \frac{\left(\lambda z_{0}\right)^{n}}{n !}\right) .
\end{aligned}
$$


Similar to the proof of Case I, we can prove that

$$
\sum_{k=0}^{m-1}\left(\prod_{i=1}^{k}\left(1+b_{i}\right) \cdot \sum_{n=m_{1}+m_{2}+\cdots+m_{k}}^{m_{1}+m_{2}+\cdots+m_{k+1}-1} \frac{\left(\lambda z_{0}\right)^{n}}{n !}\right)
$$

does not change its sign for all $t \geq t_{0}+T$.

In summary, by Case I, Case II and Theorem 3.1, all solutions of the system (1) with (2) are oscillatory in mean if and only if all solutions of the system (3) are oscillatory. The proof is complete.

Remark 3.1 Theorem 3.2 is a generalization of Theorem 2 in [11] since the condition $(\mathrm{H})$ can degenerate to the condition (C) in [11].

Theorem 3.3 Let the condition $(\mathrm{H})$ hold. If there exists a constant $\alpha>0$ such that

$$
\sum_{k=0}^{+\infty}\left(\prod_{i=1}^{k}\left|1+b_{i}\right|^{p} \cdot \sum_{n=m_{1}+m_{2}+\cdots+m_{k}}^{m_{1}+m_{2}+\cdots+m_{k+1}-1} \frac{\left(\lambda z_{0}\right)^{n}}{n !}\right) \leq \alpha e^{\lambda z_{0}}
$$

holds for all $t \geq t_{0}$, then the system (1) with (2) is (uniformly, asymptotically, uniformly asymptotically, etc.) p-moment stable if and only if the system (3) is stable correspondingly.

Proof Let $y(t)$ be any solution of the system (1) with (2). Similar to the proof of Theorem 3.1, we obtain that

$$
\mathbb{E}|y(t)|^{p}=e^{-\lambda z_{0}} \cdot \sum_{k=0}^{+\infty}\left(\prod_{i=1}^{k}\left|1+b_{i}\right|^{p} \cdot \sum_{n=m_{1}+m_{2}+\cdots+m_{k}}^{m_{1}+m_{2}+\cdots+m_{k+1}-1} \frac{\left(\lambda z_{0}\right)^{n}}{n !}\right) \cdot|x(t)|^{p} .
$$

By assumption, we obtain that

$$
\mathbb{E}|y(t)|^{p} \leq \alpha|x(t)|^{p}
$$

So, if the trivial solution of the system (3) is stable, then for any $\epsilon>0$, there exists a $\delta>0$ such that

$$
\left|x_{0}\right|<\sqrt[p]{\delta} \text { implies }|x(t)|<\sqrt[p]{\frac{\epsilon}{\alpha}} \quad \text { for all } t \geq t_{0}
$$

From $x_{0}=y_{0}$ and (8), we obtain that

$$
\left|y_{0}\right|^{p}<\delta \text { implies } \mathbb{E}|y(t)|^{p}<\epsilon \text { for all } t \geq t_{0},
$$

which means that the trivial solution of the system (1) with (2) is $p$-moment stable.

The remaining proof is similar to the proof above, so we omit it. The proof is complete.

Remark 3.2 If $b_{k}\left(\tau_{k}\right) \equiv b_{k}$ is finite for all $k=1,2, \ldots$, when the condition $(\mathrm{H})$ degenerates to the condition $(C)$ in [11], Theorem 3.3 degenerates to Theorem 3 in [11]. 


\section{Example}

Let the condition $(\mathrm{H})$ hold. Consider the second-order linear differential systems with random impulses as follows:

$$
y^{\prime \prime}(t)+t^{2} y^{\prime}(t)+y(t)=0, \quad t \in \mathbb{R}_{+}, t \neq \xi_{k}, \forall k \in \mathbb{N},
$$

and

$$
\Delta y\left(\xi_{k}\right)=b_{k} y\left(\xi_{k}^{-}\right), \quad \Delta y^{\prime}\left(\xi_{k}\right)=b_{k} y^{\prime}\left(\xi_{k}^{-}\right), \quad \forall k \in \mathbb{N},
$$

and the auxiliary differential equation

$$
x^{\prime \prime}(t)+t^{2} x^{\prime}(t)+x(t)=0 .
$$

By the classic Lyapunov's theory, the system (11) is stable, and if we let $m_{k}=1$ for $k=$ $1,2, \ldots, \lambda=1, b_{1}=1, b_{2}=-1, b_{k}$ be arbitrary for $k \geq 3, p=2, \alpha=4$, then by Theorem 3.3, we obtain that the system (9) with (10) is stable in mean square. It can be seen that for this example, the most important impulsive effects are the first two impulsive functions $b_{1}$ and $b_{2}$.

\section{Conclusion}

In this paper, we first put forward a model of second-order impulsive differential systems with Erlang distribution random impulses. Then we obtain sufficient conditions for oscillation in mean and $p$-moment stability of this model respectively. Finally, an example is presented to illustrate the efficiency of the results obtained.

\section{Competing interests}

The authors declare that they have no competing interests.

\section{Authors' contributions}

SZ completed the proof and wrote the initial draft. JS provided the problem and gave some suggestions for amendment. SZ then finalized the manuscript. Correspondence was mainly done by JS. All authors read and approved the final manuscript.

\section{Acknowledgements}

This work is supported by the NSF of China under Grant 61174039, and by the Fundamental Research Funds for the Central Universities of China.

Received: 20 September 2012 Accepted: 18 December 2012 Published: 8 January 2013

\section{References}

1. Lakshmikantham, V, Bainov, DD, Simeonov, PS: Theory of Impulsive Differential Equations. World Scientific, Singapore (1989)

2. Berezansky, L, Braverman, E: Oscillation and other properties of linear impulsive and nonimpulsive delay equations. Appl. Math. Lett. 16, 1025-1030 (2003)

3. Li, CX, Sun, JT, Sun, RY: Stability analysis of a class of stochastic differential delay equations with nonlinear impulsive effects. J. Franklin Inst. 347, 1186-1198 (2010)

4. Pan, LJ, Cao, JD: Exponential stability of impulsive stochastic functional differential equations. J. Math. Anal. Appl. 382, 672-685 (2011)

5. Chen, LJ, Sun, JT: Nonlinear boundary value problem of first order impulsive functional differential equations. J. Math. Anal. Appl. 318, 726-741 (2006)

6. Zhu, LP, Dong, QX, Li, G: Impulsive differential equations with nonlocal conditions in general Banach spaces. Adv. Differ. Equ. 2012, 10 (2012)

7. Soliman, AA: On the stability of perturbed impulsive differential systems. Appl. Math. Comput. 133, 105-117 (2002)

8. Liu, XZ: Impulsive stabilization of nonlinear systems. IMA J. Math. Control Inf. 10, 11-19 (1993)

9. Kou, CH, Zhang, SN, Wu, SJ: Stability analysis in terms of two measures for impulsive differential equations. J. Lond. Math. Soc. 66, 142-152 (2002) 
10. Pan, LJ, Cao, JD: Exponential stability of stochastic functional differential equations with Markovian switching and delayed impulses via Razumikhin method. Adv. Differ. Equ. 2012, 61 (2012)

11. Wu, SJ, Duan, YR: Oscillation, stability, and boundedness of second-order differential systems with random impulses. Comput. Math. Appl. 49, 1375-1386 (2005)

12. Wu, SJ, Meng, XZ: Boundedness of nonlinear differential systems with impulsive effect on random moments. Acta Math. Appl. Sin. 20, 147-154 (2004)

13. Wu, SJ, Guo, XL, Lin, SQ: Existence and uniqueness of solutions to random impulsive differential systems. Acta Math. Appl. Sin. 22, 595-600 (2006)

14. Anguraj, A, Wu, SJ, Vinodkumar, A: The existence and exponential stability of semilinear functional differential equations with random impulses under non-uniqueness. Nonlinear Anal. 74, 331-342 (2011)

15. Wu, SJ, Zhou, B: Existence and uniqueness of stochastic differential equations with random impulses and Markovian switching under non-Lipschitz conditions. Acta Math. Sin. Engl. Ser. 27, 519-536 (2011)

16. Ding, DR, Wang, ZD, Shen, B, Shu, HS: H-infinity state estimation for discrete-time complex networks with randomly occurring sensor saturations and randomly varying sensor delays. IEEE Trans. Neural Netw. Learn. Syst. 23, 725-736 (2012)

17. Shen, B, Wang, ZD, Liu, XH: Bounded $H$-infinity synchronization and state estimation for discrete time-varying stochastic complex networks over a finite-horizon. IEEE Trans. Neural Netw. 22, 145-157 (2011)

18. Wang, ZD, Wang, Y, Liu, DR: Global synchronization for discrete-time stochastic complex networks with randomly occurred nonlinearities and mixed time-delays. IEEE Trans. Neural Netw. 21, 11-25 (2010)

19. Csorgo, S, Hatvani, L: Stability properties of solutions of linear second order differential equations with random coefficients. J. Differ. Equ. 248, 21-49 (2010)

20. Chung, KL: A Course in Probability Theory, 3rd edn. Academic Press, San Diego (2001)

doi:10.1186/1687-1847-2013-4

Cite this article as: Zhang and Sun: Stability analysis of second-order differential systems with Erlang distribution

random impulses. Advances in Difference Equations 2013 2013:4.

\section{Submit your manuscript to a SpringerOpen ${ }^{\ominus}$ journal and benefit from:}

- Convenient online submission

- Rigorous peer review

- Immediate publication on acceptance

- Open access: articles freely available online

- High visibility within the field

- Retaining the copyright to your article 